\title{
Automatic TV Image Tracking Using Advanced Positive \\ Frame Difference Predicted Window Technique.
}

\author{
S.F.Bahgat * \\ O.El-Mowafy * \\ S.Ghoniemy *
}

\begin{abstract}
:
TV image tracking is a subject of increasing interest due to the progress of the new technologies and fabrication of high resolution long range cameras and fast microprocessors. A new technique for TV image tracking; advanced predicted window technique (APWT), is proposed. In this technique the tracking window is positioned at an advanced (predicted) position of the target moving center in a noisy background. In addition, the advanced positive frame difference predicted window technique (APFDWT) is presented, and its mathematical formulation ( including separation of moving target from noisy background, extraction of dominant features of moving target, and selection of target to be tracked ) is derived. The proposed system has the ability to identify and track close distinct targets flying in noisy stationary ambient-ground. The evaluation of target center, orientation angle, and velocity parameters are introduced to improve the tracking process.

The proposed system overcomes most of the disadvantages of the traditional TV tracking systems such as : initial manual location of the window around the selected target, initial adjustment of the window size, tracking failure due to the movement of other targets across the window, and the increased processig time due to the use of micro image processors in case of multi-target tracking. Simulation results show that the proposed tracking system is more stable and reliable
\end{abstract}

\section{Introduction :}

Recent wars have proved the effectiveness of optical sensors (TV cameras \& IR sensors) when employing electronic countermeasures against radar sensors. Computer analysis of time varying images implies the processing of a time sequence of images in order to extract some properties which change with time. There are two possible approaches to extract motion information; where the moving objects themselves are of interest (trackers typify such system), and where the motion information is used to provide additional features to scene analysis systems. Conventional TV tracking systems have some problems with target/ambient-ground separation which affects the tracking system performance. An advanced target/ambient-ground separation algorithm is proposed in this paper.

In this paper, a general description of existing TV tracking systems is summarized, and the proposed TV tracking system is introduced. The mathematical formulation of the new techniques

* Guidance Department, M.T.C., Cairo, Egypt. 
( including separation of moving targets from a noisy background, extraction of dominant features of moving targets, selection of target to be tracked, and target tracking) is presented.

Finally, system simulation including algorithmic procedures and flowcharts, obtained results, conclusion and discussion for these results is carried out.

\section{Conventional Automatic Video Tracking Systems (CAVTS) :}

The block diagram of CAVTS is shown in Fig. 1. It consists of tracking optics, which provides the image video signal to the system, video processor which uses a statistical algorithm to separate the target image pixels from the background image pixels, structural processor which is used to compute the target center and motion parameters (orientation angle, size, ......) and control processor which is used to control the camera motion to the tracking target.

A spatial band-pass filter ( window) is used to minimize the effect of the other image details. The selection and adaptation of the window size at the beginning of the tracking process is carried out manually.

G. M. Flachs [1] proposed tracking optics consisting of an interface optics and imaging subsystem to provide the capability of increasing or decreasing the image size, an electronic rotation to establish the desired object orientation, an autofocus function, and a gated image intensifier to amplify the image, and 'freeze the motion in the Field of View (FOV).

G. M. Flachs [2], [3], [4], Schalkoff [5], and Sworder [6] developed also a real time adaptive statistical clustering algorithm to separate the target image frame from the background scene at standard video rates. The basic assumption of the clustering algorithm is that the target image has some video intensities not contained in the immediate background.

The above algorithm has many disadvantages as, it can't recognize the target pixels when there is another target (stationary or moving) with the same intensity levels on the background region or when the target window is not large enough to contain a target. Again G.M.Flachs [7] and James L. Riedl [8] developped the micro image processor (MIP) which processes the data within the target window to overcome the previous disadvantages.

These TV tracking systems have many weak points, e.g. :

(1) The manual location of the target window on the FOV at the beginning of the tracking process and the selection window size especially in the multi-target tracking process.

(2) The confusion of the adaptive statistical clustering algorithm in the separation of the target from the background in the presence of other objects with the same intensity on the background region of the window.

(3) The MIP processor and the structure processor compute many structural parameters for the objects inside the window although that the objects may be stationary with the same intensity levels. In addition the window size must increase to contain all objects in the target window which increases the processing time and reduces the measurement resolution.

To overcome these problems, this paper proposes the following modifications :

(1) Automatic location and parameter calculation of all moving targets.

(2) Automatic selection of target window specifications. 


\section{Proposed Automatic Video Tracking System (PAVTS ) [9] :}

\subsection{Proposed system description :}

The proposed tracking system that tracks a moving target in noisy ambient-ground (background and foreground) is designed assuming :

(1) No overlapping and suitable separation between targets at the beginning of targets detection.

(2) Moving targets have higher intensity levels than the surrounding ambient-ground pixels.

The proposed system is a modification of the conventional video tracking system according

to Fig. 2. The video processor receives the video signal from the TV camera, and converts it into binary data to be stored in dynamic RAMs and updated continuously. The introduced advanced target ambient-ground separation (ATAGS) is responsible for manipulating the stored data, calculating the frame difference (FD), and determining the moving objects intensity distribution to separate the moving target from stationary background. The positive difference of the result, known as positive frame difference (PFD), helps to recognize the direction of target motion. The target selection is responsible for specifying the target to be tracked. The tracking processor is responsible for tracking the selected target .

\subsection{Analysis and Formulation :}

The proposed system determines the intensity levels distribution of the moving objects and the closed ambient-ground pixels, by computing the FD of two sequential frames, and generates a positive frame difference binary mask (PFDBM). The PFDBM is pointing at the pixels affected by the objects motion in the FOV. Using it and referring to the two real frames, one gives the target intensity distribution and other gives the ambient-ground distribution. So the system is able to recognize the targets pixels from the closed ambient-ground pixels without confusion.

In the real time frame a scan window image technique (SWIT) is used to select the moving objects, as follows:

(1) A $6 \times 6$ window is located around the pointer of the target pixels.

(2) Using an iterative method, the system expands the window size until it completely covers the target.

(3) The detected moving object data (position and size) is stored.

(4) The system eliminates its pointer pixels from the PFDBM.

(5) The system continues scanning the PFDBM, until the recognition of all the moving objects in the FOV.

The relative velocity of the moving objects per frame is evaluated by repeating the whole previous operations for the next processing frame. In the proposed system, the selection of the tracked target can be either automatic or semi-automatic. In semi-automatic case, a symbol like $(+)$ is manually adjusted at target center $(x, y)$ and the system compares this position with the pre-determined positions of all targets and displays the parameters of the selected target. Then the system starts the tracking process. In automatic case, the system compares between the predetermined parameters of the required target and the measured parameters of all targets in the FOV. Then the system selects the closest target, and directs the window to start the tracking process. 
The tracking process is the heart of the TV tracking operation. The tracking processor receives the selected target parameters (position, size, intensity distribution, and relative velocity), generates the tracking window, and directs it to the selected target. The processor determines the new position of the target continuously and directs the tracking window to follow the target by decreasing the error between the center of the selected target and the center of the tracking window. The system repeats this process continuously to perform the automatic tracking. This technique was found to give better results, when the system updates the changes in target intensity distributions.

The adaptive window positive frame technique is proposed to measure the target intensity distribution, by locating the forward target pixels inside the tracking window. This can be carried out using a subtractor producing the frame difference between the window pixels in the two successive frames. A comparator is used to eliminate the pixels that have difference levels less than a threshold value, to minimize the noise effect.

\section{Mathematical formulation for the ATAGS:}

\subsection{Extraction of Moving Objects:}

A general two-dimensional time-varying noiseless scene $\mathrm{S}$ consisting of the time-varying object Q, overlapping the background scene B, and being overlapped by the foreground scene $\mathrm{F}$ at time $\mathrm{t}_{1}$ may be expressed as follows:

$$
\mathrm{S}\left(\mathrm{x}, \mathrm{y} ; \mathrm{t}_{1}\right)=\mathrm{F}(\mathrm{x}, \mathrm{y})+\left[1-\mathrm{U}(\mathrm{F}(\mathrm{x}, \mathrm{y})) \mid \sum_{i=1}^{N} \mathrm{Q}_{i}\left(\mathrm{x}, \mathrm{y} ; \mathrm{t}_{1}\right)+[1-\mathrm{U}(\mathrm{F}(\mathrm{x}, \mathrm{y}))] \prod_{i=1}^{N}\left[1-\mathrm{U}\left(\mathrm{Q}_{\mathrm{i}}\left(\mathrm{x}, \mathrm{y} ; \mathrm{t}_{1}\right)\right)\right] \mathrm{B}(\mathrm{x}, \mathrm{y})\right.
$$

Where: $(x, y)$ is the pixel position in the image frame

$$
\begin{array}{rlrl}
\mathrm{U}(\mathrm{F}(\mathrm{x}, \mathrm{y})) & =" 1 " & & \text { if } \mathrm{F}(\mathrm{x}, \mathrm{y})>0 \\
& =" 0 " & \text { if } \mathrm{F}(\mathrm{x}, \mathrm{y})<0
\end{array}
$$

Under the assumption that there is no overlapping between the moving objects, we can show that for any pixel $(x, y)$ belonging to $Q_{S}, Q_{i}(x, y ; t)=0$ for all $i$ except at $i=s$.

then:

$$
\prod_{i=1}^{N}\left[1-U\left(Q_{i}(x, y ; t)\right)\right]=\left[1-U\left(Q_{s}(x, y ; t)\right)\right]
$$

also

$$
\left[1-U\left(\sum_{i=1}^{N} Q_{i}(x, y ; t)\right)\right]=\left[1-U\left(Q_{s}(x, y ; t)\right)\right]
$$

From (2) - (3) and substituting in (1) we get :

$$
\mathrm{S}\left(\mathrm{x}, \mathrm{y} ; \mathrm{t}_{1}\right)=\mathrm{F}(\mathrm{x}, \mathrm{y})+[1-\mathrm{U}(\mathrm{F}(\mathrm{x}, \mathrm{y}))] \sum_{i=1}^{N} \mathrm{Q}_{i}\left(\mathrm{x}, \mathrm{y} ; \mathrm{t}_{1}\right)+[1-\mathrm{U}(\mathrm{F}(\mathrm{x}, \mathrm{y}))]\left[1-\mathrm{U}\left(\sum_{i=1}^{N} \mathrm{Q}_{i}\left(\mathrm{x}, \mathrm{y} ; \mathrm{t}_{1}\right)\right)\right] \mathrm{B}(\mathrm{x}, \mathrm{y})
$$

similarly at time $\mathrm{t}_{2}$

$$
\mathrm{S}\left(\mathrm{x}, \mathrm{y} ; \mathrm{t}_{2}\right)=\mathrm{F}(\mathrm{x}, \mathrm{y})+[1-\mathrm{U}(\mathrm{F}(\mathrm{x}, \mathrm{y}))] \sum_{\mathrm{i}=1}^{N} \mathrm{Q}_{\mathrm{i}}\left(\mathrm{x}, \mathrm{y} ; \mathrm{t}_{2}\right)+[1-\mathrm{U}(\mathrm{F}(\mathrm{x}, \mathrm{y}))]\left[1-\mathrm{U}\left(\sum_{\mathrm{i}=1}^{\mathrm{N}} \mathrm{Q}_{\mathrm{i}}\left(\mathrm{x}, \mathrm{y} ; \mathrm{t}_{2}\right)\right)\right] \mathrm{B}(\mathrm{x}, \mathrm{y})
$$


The frame difference FD is expressed as

$$
\begin{aligned}
F D= & S\left(x, y ; t_{2}\right)-S\left(x, y ; t_{1}\right) \\
F D= & {[1-U(F(x, y))]\left[\sum_{i=1}^{N} Q_{i}\left(x, y ; t_{2}\right)-\sum_{i=1}^{N} Q_{i}\left(x, y ; t_{1}\right)\right] } \\
& +[1-U(F(x, y))]\left[U\left(\sum_{i=1}^{N} Q_{i}\left(x, y ; t_{1}\right)\right)-U\left(\sum_{i=1}^{N} Q_{i}\left(x, y ; t_{2}\right)\right)\right] B(x, y)
\end{aligned}
$$

A subtractor is used to produce the FD, and a comparator is used to eliminate the absolute intensity difference levels less than a threshold value. The proposed system needs one mask which refers to the targets pixels in one frame and the ambient-ground in the other frame. The needed mask is formed by using the positive frame difference (PFD), which marks only the moving object forward pixels, and is expressed as:

$$
\operatorname{PFD}=[1-\mathrm{U}(\mathrm{F}(\mathrm{x}, \mathrm{y}))]\left[1-\mathrm{U}\left(\sum_{\mathrm{i}=1}^{\mathrm{N}} \mathrm{Q}_{\mathrm{i}}\left(\mathrm{x}, \mathrm{y} ; \mathrm{t}_{1}\right)\right)\right] \mathrm{U}\left(\sum_{\mathrm{i}=1}^{\mathrm{N}} \mathrm{Q}_{\mathrm{i}}\left(\mathrm{x}, \mathrm{y} ; \mathrm{t}_{2}\right)\right)\left\{\sum_{\mathrm{i}=1}^{\mathrm{N}} \mathrm{Q}_{\mathrm{i}}\left(\mathrm{x}, \mathrm{y} ; \mathrm{t}_{2}\right)-\mathrm{B}(\mathrm{x}, \mathrm{y})\right\}
$$

The PFD is used to produce the positive frame difference binary mask (PFDBM) as U(PFD). Using PFDBM and the frame of time $t_{1}$, the system can extract the background distribution intensity by scanning PFDBM $\left\{\mathrm{S}\left(\mathrm{x}, \mathrm{y} ; \mathrm{t}_{1}\right)\right\}$. Also using the PFDBM and the frame at time $\mathrm{t}_{2}$, the system can extract the moving objects intensity distribution by scanning PFDBM $\left\{\mathrm{S}\left(\mathrm{x}, \mathrm{y} ; \mathrm{t}_{2}\right)\right\}$. This distribution is used to classify each pixel as target or ambient-ground pixel. The intensity distribution for the moving targets $\mathrm{h}^{\mathrm{T}}$ and for the ambient-ground distribution $\mathrm{h}^{\mathrm{A}}$ can be expressed as follows:

$$
\mathrm{h}^{\mathrm{T}}(\mathrm{i})=\Sigma \text { PFDBM } \mathrm{T}\left\{\mathrm{i}-\mathrm{S}\left(\mathrm{x}, \mathrm{y} ; \mathrm{t}_{2}\right)\right\}
$$

and

$$
\mathrm{h}^{\mathrm{A}}(\mathrm{i})=\Sigma \text { PFDBM } \mathrm{T}\left\{\mathrm{i}-\mathrm{S}\left(\mathrm{x}, \mathrm{y} ; \mathrm{t}_{1}\right)\right\}
$$

where

$$
\begin{aligned}
\mathrm{T}\{\mathrm{i}-\mathrm{k}\}=0 & & \text { if } & \mathrm{k} \neq \mathrm{i} \\
& =1 & & \text { if } \quad \mathrm{k}=\mathrm{i}
\end{aligned}
$$

for all gray levels $[0, d]$

To prevent any confusion due to the common intensity levels in $\mathrm{h}^{\mathrm{T}}$ and $\mathrm{h}^{\mathrm{A}}$ the system eliminates these levels from the targets intensity distribution.

The separation of the moving objects in the FOV is carried out using the PFDBM as a pointer of objects pixels in the last frame and using a $3 \times 3$ noise elimination window over that pixel to identify it as target or noise. The pixel is identified as a target, if the number of pixels inside the window exceeds the threshold value. The obtained pixel is considered as a pixel of a moving object and using the estimated target pixels intensity distribution we can determine the target center and size. This is achieved using the scan window image technique (SWIT).

\subsection{Features of Moving Targets :}

SWIT is used to compute the target center and the optimum window size to cover it. In this technique the system locates a $6 \times 6$ window around the predetermined target pixel, to 
compute the $\mathrm{x}$ and $\mathrm{y}$ border coordinates of the target inside the window $\left(\mathrm{X}_{\max }, \mathrm{Y}_{\max }, \mathrm{X}_{\min }\right.$, $\left.\mathrm{Y}_{\min }\right)$. The center of the target is expressed as:

$$
\mathrm{X}_{\mathrm{C}_{\mathrm{i}}}=\frac{\mathrm{X}_{\max }-\mathrm{X}_{\text {min }}}{2}, \quad \mathrm{Y}_{\mathrm{c}_{\mathrm{i}}}=\frac{\mathrm{Y}_{\text {max }}-\mathrm{Y}_{\min }}{2}
$$

the size of the target is expressed as:

$$
\mathrm{Sz}_{\mathrm{i}}=\sum_{\mathrm{x}, \mathrm{y}} \mathrm{SW}_{\mathrm{i}} \mathrm{U}\left(\mathrm{Q}_{\mathrm{i}}(\mathrm{x}, \mathrm{y} ; \mathrm{t}+1)\right.
$$

where

$$
\begin{aligned}
\mathrm{SW}_{\mathrm{i}} & =" 1 " & & \text { if }(\mathrm{x}, \mathrm{y}) \text { is inside the SWIT window } \\
& =" 0 " & & \text { if }(\mathrm{x}, \mathrm{y}) \text { is outside the SWIT window }
\end{aligned}
$$

The dimensions of the window $\left(\mathrm{x}_{\mathrm{W}}\right.$ and $\left.\mathrm{ly}_{\mathrm{W}}\right)$ are determined as follows:

$$
1 x_{W}=\left(X_{\max }-X_{\min }\right)+4, \quad \operatorname{ly}_{W}=\left(Y_{\max }-Y_{\min }\right)+4
$$

The system locates the new window at the new center, and recomputes $\mathrm{Xc}_{\mathrm{i}}, \mathrm{Yc}_{\mathrm{i}}, \mathrm{Sz}_{\mathrm{i}}, \mathrm{Ix}_{\mathrm{W}}$, and $\mathrm{ly}_{\mathrm{w}}$. If $\mathrm{Sz}_{\mathrm{i}}$ changes, the system increases the window size (lx, ly) and repeats the previous process again until having two successive target sizes with the same value, indicating that the window contains all the target pixels.

Then the specific target and ambient-ground intensity distributions can be written as:

$$
\begin{aligned}
& h_{\mathrm{s}}^{\mathrm{T}}(\mathrm{i})=\sum \mathrm{SW}_{\mathrm{S}} \text { PFDBM T }\left\{\mathrm{i}-\mathrm{S}\left(\mathrm{x}, \mathrm{y} ; \mathrm{t}_{2}\right)\right\} \\
& h_{s}^{\wedge}(i)=\sum S_{S} \text { PFDBM T }\left\{i-S\left(x, y ; t_{1}\right)\right\} \\
& \text { where } \quad \mathrm{SW}_{\mathrm{S}} \text { is the target predictor window } \\
& \mathrm{s} \quad \text { is the index of the target }
\end{aligned}
$$

The process for generating the window on the moving target using SWIT process is shown in Fig. 3.

To recognize the other moving objects, the system eliminates the previous target pointer pixels from the PFDBM, using ([1-SW $]$ PFDBM) as a new mask. The scanning of the new mask continues to find out any other moving object in the [1-SW 1 PFDBM. The mask can be generally expressed as follows:

$$
\text { WPFDBM }=\left[1-\sum_{i=0}^{N} S_{i}\right] \text { PFDBM }
$$

where : $\mathrm{N}$ is the number of moving objects inside the FOV.

A flowchart for multi-targets separation from a noisy ambient-ground in a FOV image frame is shown in Fig.4.

The advantages of the proposed targets/ambient-ground separation are: the ability to recognize targets (moving objects) in a very noisy ambient-ground, and the ability to overcome the disadvantages of the conventional statistical clustering separation algorithm, (initial manual location window size adjustment,...) .

\section{4-3 Target Selection:}

The selection of the target to be tracked can be established automatically or semiautomatically. The semi-automatic selection is provided using two drivers to produce voltage signals for adjusting a cross-hairs symbol $(+)$ at the center of the moving target. 
The $(x$ and $y)$ values are used to determine the address of the location of the symbol in the FOV frame.

It is mentioned in the ATAGS, that targets are recognized using the SWIT, in which the window that contains the target is specified by comparing the selected manual location by the windows pixels location, it can recognize to what window that manual location belongs, and then starts the tracking process. This can be done again for another targets in a parallel tracking process.

The semi-automatic selection can also be achieved by displaying the previous measured features of each target and then selecting the assigned one, but this method takes long time and is not reliable.

The automatic selection is carried out by comparing the measured feature vectors with a specific stored feature vector (Xc, Yc, Sz, Intensity distribution), and selecting the nearest neighbor. For example, if the system is interested in

the target with minimum displacement from the center of FOV, the system computes the displacement of each target center from the FOV center expressed as:

$$
\mathrm{d}_{\mathrm{i}}=\sqrt{\left(\mathrm{X}_{\mathrm{c}_{i}}-\mathrm{X}_{\mathrm{f}}\right)^{2}+\left(\mathrm{Y}_{\mathrm{c}_{i}}-\mathrm{Y}_{\mathrm{f}}\right)^{2}}
$$

where $\quad X_{f}$ and $Y_{f}$ are the coordinates of center location of the FOV

and selecting the target with the minimum $d_{i}$, then locating the pre-computed window around it and starting the tracking process.

Also the system can select the target with the biggest or smallest size, the target with the highest or lowest relative velocity. It can also use the intensity distribution of each target as a selection feature. Generally the system can select a class of targets in order.

\subsection{Target Tracking:}

The accuracy and stability of the tracking process are the main objectives of tracking system. The use of a band-pass filter (window) minimizes the effect of the parasitic disturbance due to the ambient-ground or other moving objects.

The window has to be wide enough to cover the object and its motion. On the other hand, it must be narrow enough for minimizing the processing time, increasing the measured parameters accuracy, and decreasing the effect of other environment. The system computes the center of each target inside its window as follows:

$$
\begin{aligned}
& X_{c}=\frac{\operatorname{TWs} \sum_{x, y} \sum_{i=1}^{N} U\left(Q_{i}(x, y ; t)\right) x}{T W s \sum_{x, y} \sum_{i=1}^{N} U\left(Q_{i}(x, y ; t)\right)} \\
& Y_{C}=\frac{\operatorname{TWs} \sum_{x, y} \sum_{i=1}^{N} U\left(Q_{i}(x, y ; t)\right) y}{\operatorname{TWs} \sum_{x, y} \sum_{i=1}^{N} U\left(Q_{i}(x, y ; t)\right)}
\end{aligned}
$$

where: 


$$
\begin{aligned}
& \text { TWs }=" 1 " \quad \text { if pixel }(\mathrm{x}, \mathrm{y}) \in \text { the target window } \\
& =" 0 " \quad \text { if pixel }(\mathrm{x}, \mathrm{y}) \notin \text { the target window } \\
& \text { and } \mathrm{s} \quad \text { is the target mark number }
\end{aligned}
$$

The selection of the target pixels can be achieved by updating the target and closed ambient-ground intensity distributions with time. An advanced positive frame difference window technique is used to determine the target intensity distribution inside the tracking window.

The system uses a subtractor to obtain the window frame difference between the new window pixels and the same pixels in the previous frame. Also, the system uses a comparator to eliminate the noise pixels caused by any perturbation in the FOV image frame. Let W(x,y;t 1$)$ represent the window pixels at time $t_{1}$ then:

$$
\mathrm{W}\left(\mathrm{x}, \mathrm{y} ; \mathrm{t}_{1}\right)=\mathrm{TW}_{\mathrm{S}} \mathrm{S}\left(\mathrm{x}, \mathrm{y} ; \mathrm{t}_{1}\right)
$$

the window frame difference WFD can be expressed as:

$$
\begin{aligned}
\mathbf{W F D}= & \mathbf{W}\left(\mathbf{x}, \mathbf{y} ; \mathbf{t}_{2}\right)-\mathbf{W}\left(\mathbf{x}, \mathbf{y} ; \mathbf{t}_{\mathbf{1}}\right) \\
= & \mathbf{T W}_{\mathbf{s}}\left\{\mathbf{S}\left(\mathbf{x}, \mathbf{y} ; \mathbf{t}_{\mathbf{2}}\right)-\mathbf{S}\left(\mathbf{x}, \mathbf{y} ; \mathbf{t}_{\mathbf{1}}\right)\right\} \\
= & \mathbf{T W}[1-U(F(x, y))]\}\left[\sum_{i=1}^{N} Q_{i}\left(x, y ; t_{2}\right)-\sum_{i=1}^{N} Q_{i}\left(x, y ; t_{1}\right)\right] \\
& +[1-U(F(x, y))]\left[U\left(\sum_{i=1}^{N}\left(Q_{i}\left(x, y ; t_{1}\right)\right)-U\left(\sum_{i=1}^{N} Q_{i}\left(x, y ; t_{2}\right)\right)\right] B(x, y)\right\}
\end{aligned}
$$

The system is interested in the target forward pixels, so using a comparator, it produces the window positive frame difference (WPFD) which is expressed as:

$$
\begin{gathered}
\text { WPFD }=\mathbf{T W}_{\mathbf{S}}[1-U(F(x, y))]\left[1-U\left(\sum_{i=1}^{N} Q_{i}\left(x, y ; t_{1}\right)\right)\right] U\left(\sum_{i=1}^{N} Q_{i}\left(x, y ; t_{2}\right)\right) \\
\left\{\sum_{i=1}^{N} Q_{i}\left(x, y ; t_{2}\right)-B(x, y)\right\}
\end{gathered}
$$

Using the WPFD the system produces a mask called WPFD binary mask, denoted as $\mathrm{U}(\mathrm{WPFD})$, to compute the target pixels intensity distribution $h_{\mathrm{S}}^{\mathrm{T}}(\mathrm{i})$ from TW $S\left(x, y ; t_{2}\right)$ and the ambient-ground pixels distribution $h_{S}^{\wedge}(i)$ from TW $S\left(x, y ; t_{1}\right)$ as follows:

$$
\begin{array}{llll} 
& \mathrm{h}_{\mathrm{S}} \mathrm{T}(\mathrm{i}) & =\sum \text { WPFDBM T }\left\{\mathrm{i}-\mathrm{S}\left(\mathrm{x}, \mathrm{y} ; \mathrm{t}_{2}\right)\right\} \\
\text { where } & \mathrm{h}_{\mathrm{S}} \mathrm{A}_{(\mathrm{i})} & =\Sigma \text { WPFDBM T }\left\{\mathrm{i}-\mathrm{S}\left(\mathrm{x}, \mathrm{y} ; \mathrm{t}_{1}\right)\right\} \\
& \mathrm{T}\{\mathrm{i}-\mathrm{k}\} & =0 & \text { if } \mathrm{k} \neq \mathrm{i} \\
& & =1 & \text { if } \mathrm{k}=\mathrm{i},\{\mathrm{i}=0,1, \ldots, \mathrm{d}\}
\end{array}
$$

The common intensity levels in the target intensity distribution and the ambientground intensity distribution are eliminated from target intensity distribution. The WPFD can be used with a constant size window, independent of target size, and still dependable. The narrowing of the window size minimizes the effect of the other (moving or constant) objects having the same intensity levels. Figure 5 a) shows the window in the previous real frame, b) shows the window in the updated real frame and c) shows the real implementation of the WPFD, and how it marks the forward target pixels and few noise pixels due to the perturbation inside the window only.

It uses the new distribution (22) to recognize the target pixels, and using the formula in equations (17) and (18), to computes the target's center and continue the tracking process. Figure 6 shows how the window tracks the target, by using the WPFD tracking process.

The system can use a projection process to compute the target orientation angle, to create a parallelogram window with slope angle equal to the orientation angle measured in the previous processing frame, to improve the filteration of the noise and other objects. Fig. 7 
shows the tracking of a simulated target and how the window parallelogram changes with the orientation angle.

The system can separate the selected moving target and measure its structural parameters, but if the moving target overlaps other moving or constant objects with the same intensity distribution, the system will be confused, and the window will be expanded to cover the moving target and the overlapping objects as shown in figure 8 . This widening increases the processing time and degrades the system accuracy. When the overlapping is over, the system doesn't know which is the selected target, so it will track the two objects, without being differentiated.

The system can eliminate the effect of the constant objects absolutely by tracking only the forward target pixels (the pixels having maximum positive intensity difference), the modified formula of the target center is:

$$
\begin{gathered}
X_{c}=\frac{\sum_{x, y} U(\operatorname{WPFD}(x, y ; t)) x}{\sum_{x, y} U(\operatorname{WPFD}(x, y ; t))} \\
Y_{c}=\frac{\sum_{x, y} U(\operatorname{WPFD}(x, y ; t)) y}{\sum_{x, y} U(\operatorname{WPFD}(x, y ; t))}
\end{gathered}
$$

During the tracking process using equations (24) and (25), the background pixels will be eliminated and so its effect. But the system cannot use MIP, because the PFD contains only the forward moving object pixels which may be a collection of clustered pixels, one moving object may have one or more cluster pixels, so the MIP will count each cluster as a moving target. In this case, the system can not estimate the moving target size, and also the needed size of the window.

The system will track the selected target at the beginning using the window and the MIP process, to separate the target and adjust the window size, after that the window will be fixed and the system tracks the forward moving target pixels as described in equations (24) and (25).

The main disadvantage of the conventional video tracking systems is the confusion of the target background seperation process due to the existance of other objects with the same intensity feature in background window region. This problem is overcomed in our proposed system by using positive frame difference technique that can separate the moving object in very noisy FOV. In addition window frame difference technique is applied to separate the target pixels levels inside the window.

\section{Conclusion and Future Work :}

The conventional TV tracking system uses a statistical clustering algorithm for target $\backslash$ background separation. This technique requires initial manual location, and size adjustment of the window around the target. In addition, the tracking process fails due to the cross movement of any object having the same intensity (moving or fixed) in the background region.

The proposed system has the ability to identify and track close distinct targets flying in a noisy stationary ambient- ground. The evaluation of the target center, orientation angle, and its velocity parameters are introduced to improve the tracking process. In addition, the proposed 
system overcomes most of the disadvantges of the traditional TV tracking system as mentioned before. Simulation results show that the proposed tracking system is more stable and reliable.

The effect of the restricted window size appears when the target size increases more than the window size ( due to its approaching motion ). In this case the measured displacement of the target center is not the true displacement because the window measures the center of the target pixels inside it only. Then the tracking process will fail. So the window size has to be with big size to contain the object and its motion. But the increase of the window will maximize the effect of the other moving objects. The system has to get a tracking method minimizing the window size and still measuring a closed target center in the case of approaching target.

To be close to the target motion with restricted window size, the system has to predict the next position of the moving target in the next frame and locate the window

at predicted position. This will minimize the displacement between the target center and the window center, so the system can track the target because the prediction restricts the displacement within the window. The tracking using predictors will be considered as future work.

\section{REFERENCES :}

[1] Gerald M. Flachs, et al.,"A Structure and Dynamatical Model of a Real VideoTracking System", Proc. of NAECON pp. 169-172, 1976

[2] Gerald M. Flachs, et al.,"A Real-Time Structural Tracking Agorithm", Proc. of NAECON pp. 161-168, 1976

[3] Gerald M. Flachs, et al., "A Pre-Protoype Real-Time Video Tracking Agorithm", Proc. of NAECON pp. 156-160, 1976

[4] Gerald M. Flachs, et al.,"A Rreal-Time Video Tracking System", IEEE Transactions on Pattern Analysis and Machine Intelligence, Vol. Pami-2, No 1, pp. 47-56, January 1980.

[5] Schalkoff,R.J. and McVey,E.S.," A Model and Tracking Algorithm for a Class of Video Targets," IEEE Trans. PAMI, Vol PAMI-4, No.1, pp.2-10, 1982.

[6] D.D.Sworder, et al," Gain Adaptive Tracking," Journal of Guidance, Control and Dynamics, Vol.16, No.5, September 1993.

[7] Gerald M. Flachs, et al.,"An Automatic Video Tracking System", Proc. of NAECON '77, pp. 361-368, 1977.

[8] James L. Riedl, "CCD Sensor and Microprocessor Application to Missile Tracking", SPIE Vol. 95 Modern Utilization of Infrared Technology II pp. 148-154, 1976.

[9] Osama M.El-Mowafy," Image Processing For TV Image Tracking," M.Sc. thesis, Military Technical College, cairo, December 1994. 


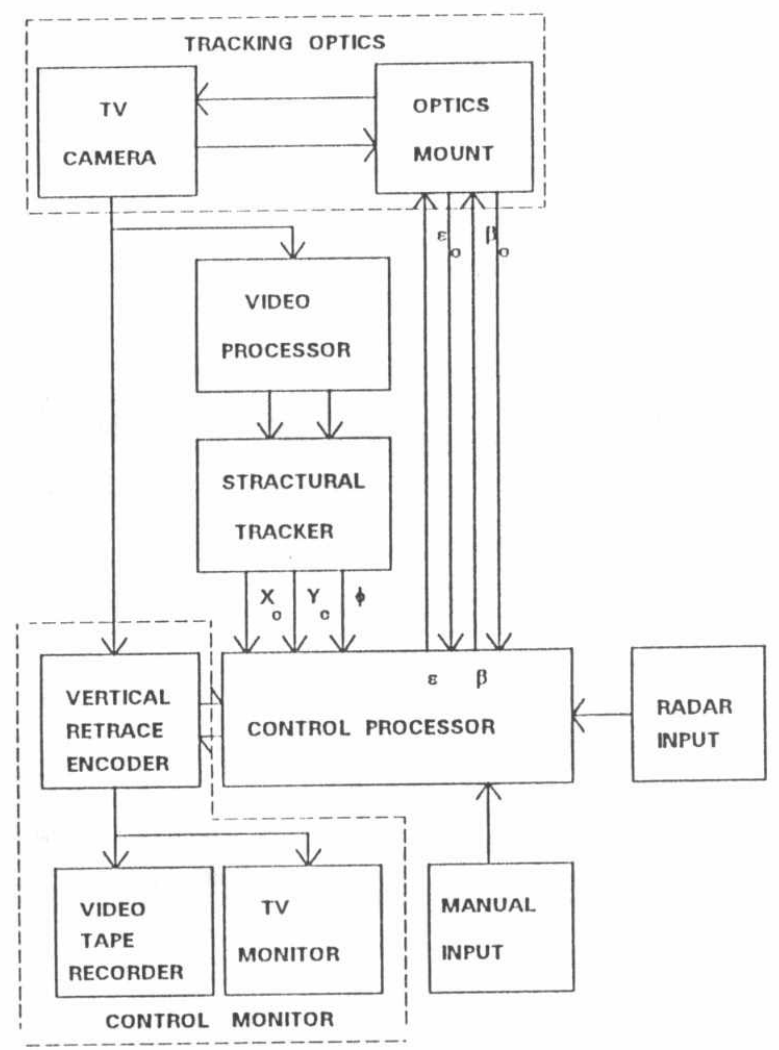

Fig. 1. A block diagram for the CAVTS

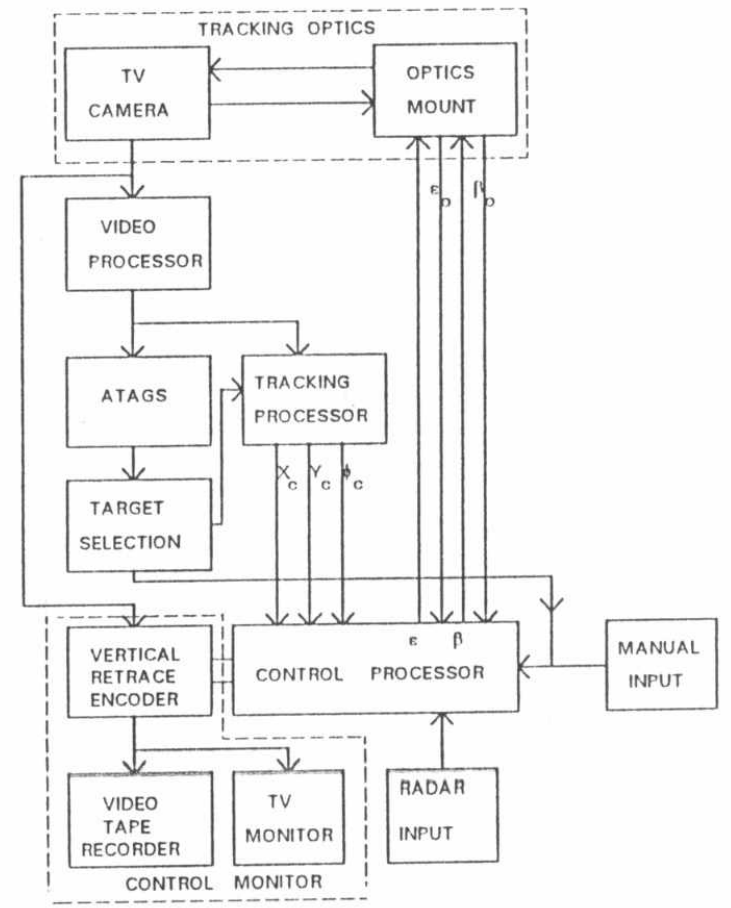

Fig. 2. A block diagram for the PAVTS 


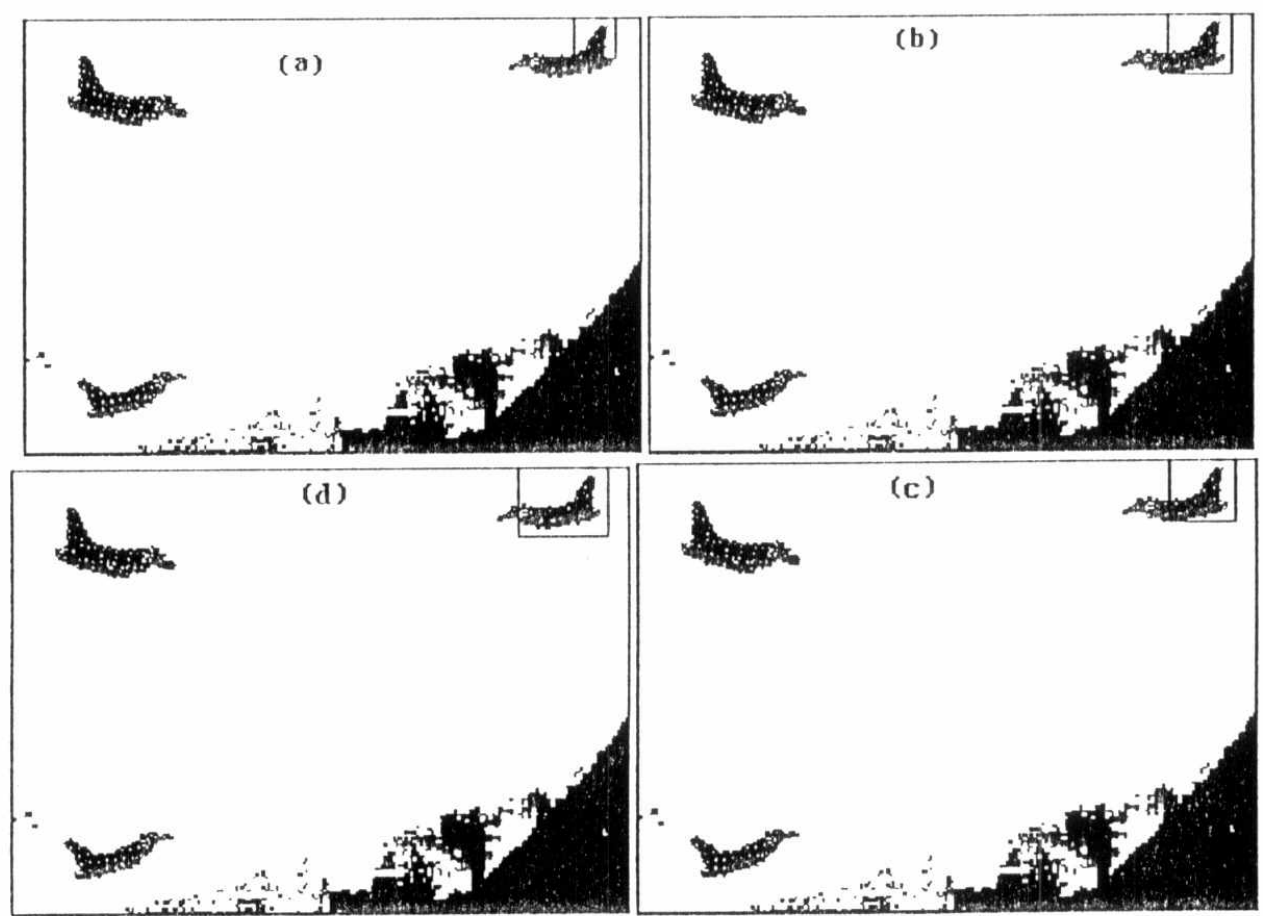

Fig. 3. The generation of a window around the moving target using SWIT.

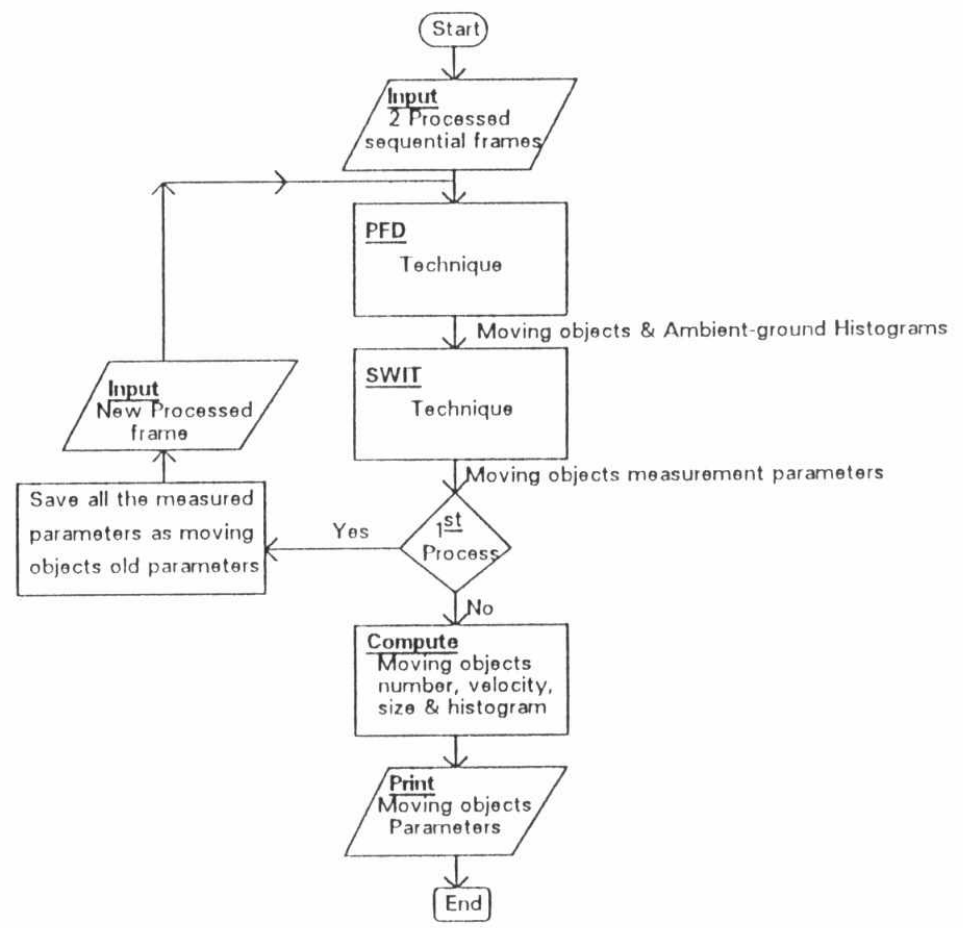

Fig. 4. Flowchart for multi-target separation from a noisy ambient-ground in FOV image Frame. 


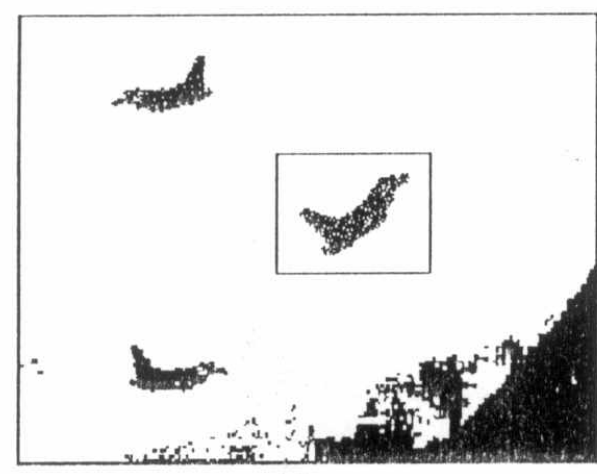

(a)

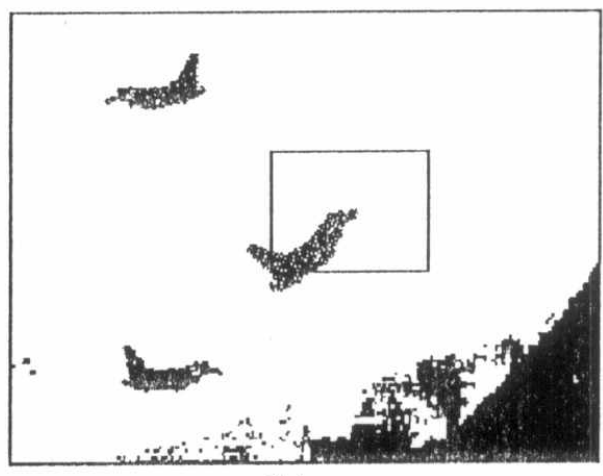

(b)

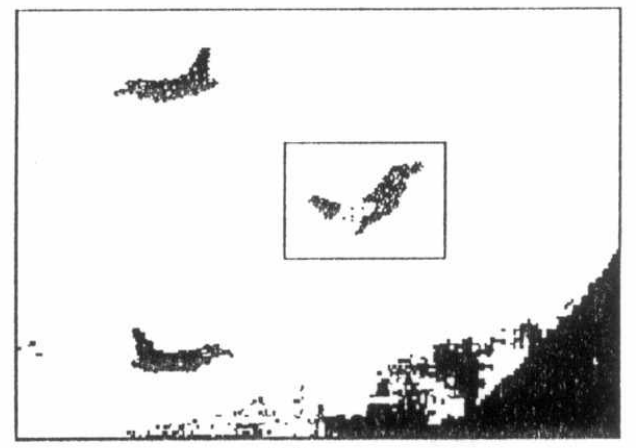

(c)

Fig. 5. WPFD Process.
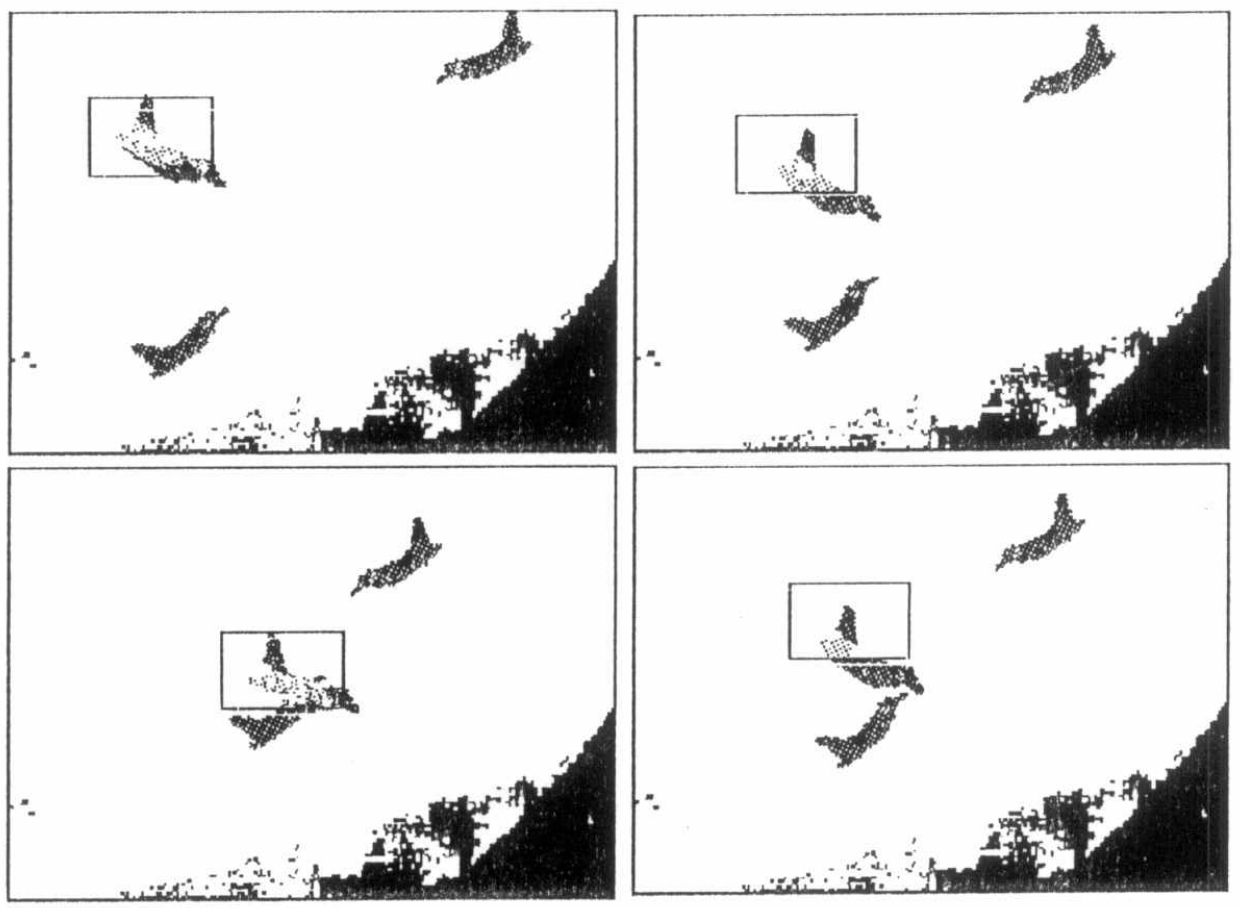

Fig. 6. The WPFD tracking process. 


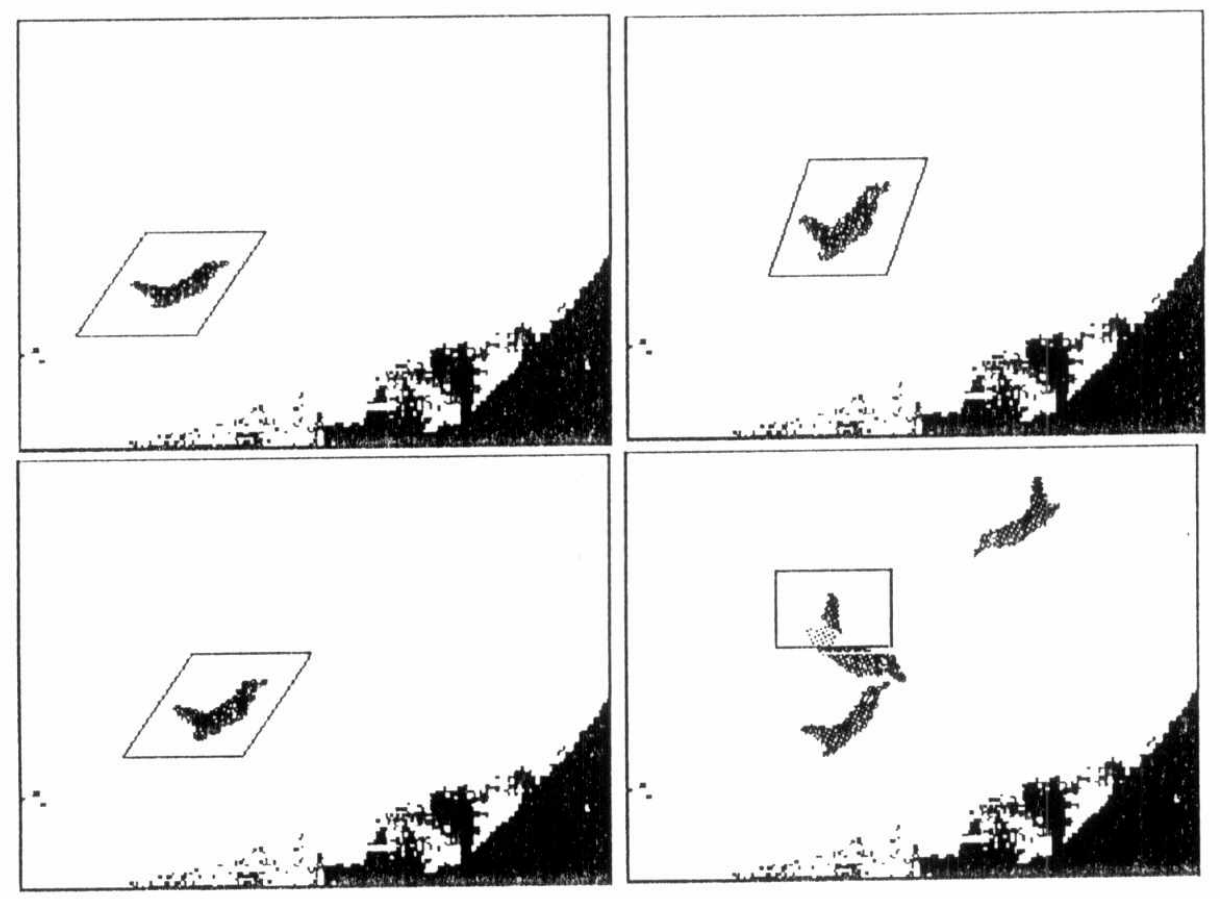

Fig. 7. Tracking process (using a parallelogram window).
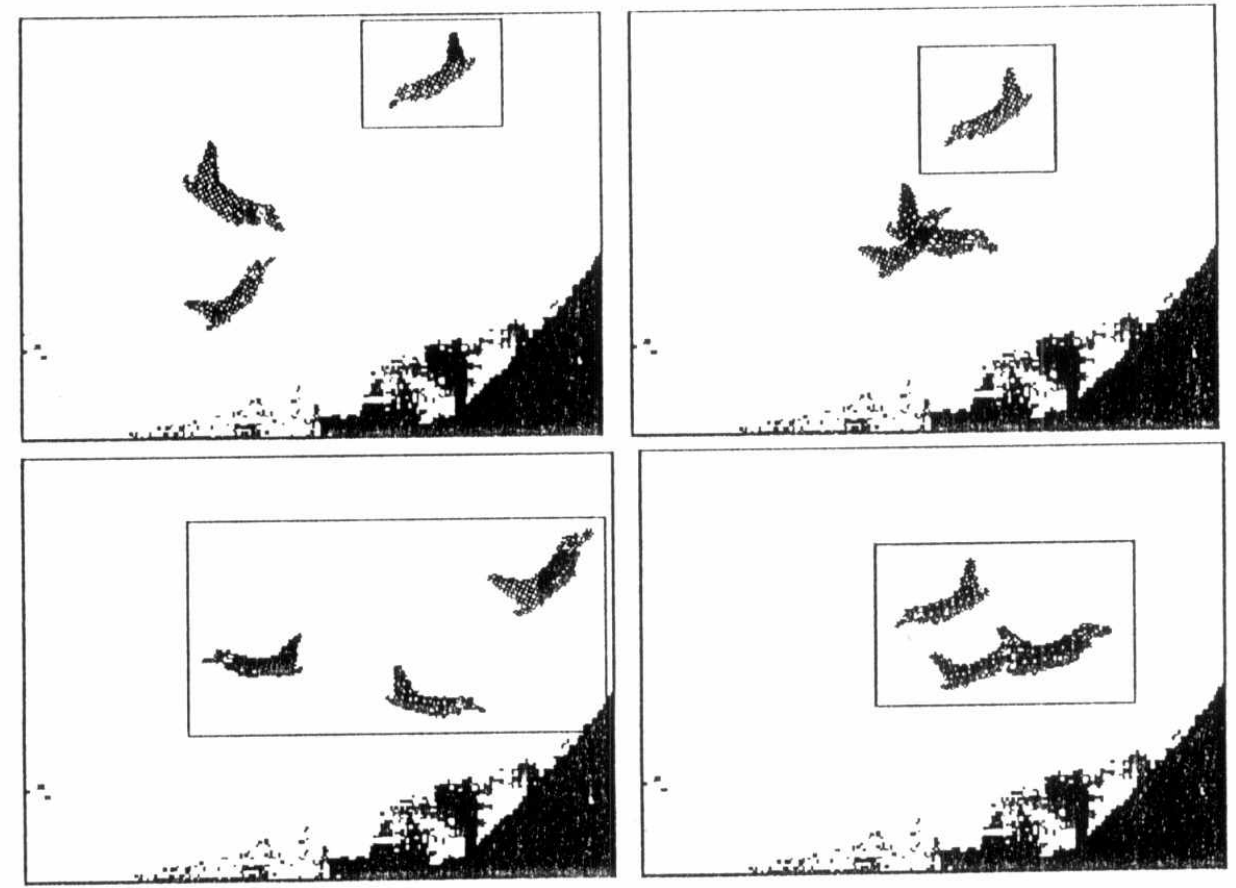

Fig. 8. Expanded window tracking due to crossing object using the MIP process . 